Research Paper

\title{
Anatomical versus Non-anatomical Resection for Hepatocellular Carcinoma with Microscope Vascular Invasion: A Propensity Score Matching Analysis
}

\author{
Xiao-Ping Zhong1 ${ }^{*}$, Yong-Fa Zhang ${ }^{3}$, Jie Mei², Shao-Hua Li², Anna Kan², Liang-He Lu², Min-Shan Chen², \\ Wei Wei ${ }^{\circledR}$, Rong-Ping Guo ${ }^{2 \bowtie}$ \\ 1. Department of Burn and Plastic Surgery, 2nd Affiliated Hospital of Shantou University Medical College, Shantou 515041, China; \\ 2. Department of Hepatobiliary Oncology of Sun Yat-sen University Cancer Center; State Key Laboratory of Oncology in South China; Collaborative \\ Innovation Center for Cancer Medicine, Guangzhou 510060, China; \\ 3. Department of Hepatic Surgery, Fudan University Shanghai Cancer Center, Shanghai, China; Department of Oncology, Shanghai Medical College, Fudan \\ University, Shanghai 200032, China. \\ *These authors contribute equally to this study.
}

$\triangle$ Corresponding author: Rong-Ping Guo, M.D. Department of Hepatobiliary Oncology, Sun Yat-sen University Cancer Center, 651 Dongfeng Road East, Guangzhou, 510060, People's Republic of China. Telephone: (8620)-87342266; Fax: (8620)-87342266; Email: guorp@sysucc.org.cn; Wei Wei, M.D. Department of Hepatobiliary Oncology, Sun Yat-sen University Cancer Center, 651 Dongfeng Road East, Guangzhou, 510060, People's Republic of China. Telephone: (8620)-87343115; Fax: (8620)-87343115; Email: weiwei@sysu.edu.cn

(c) The author(s). This is an open access article distributed under the terms of the Creative Commons Attribution License (https://creativecommons.org/licenses/by/4.0/). See http://ivyspring.com/terms for full terms and conditions.

Received: 2018.12.28; Accepted: 2019.06.01; Published: 2019.07.05

\begin{abstract}
Background: The benefits of anatomical resection (AR) and non-anatomical resection (NAR) on hepatocellular carcinoma $(\mathrm{HCC})$ patients with microscope vascular invasion (MVI) remain unknown. We aimed to investigate the prognostic outcomes of AR and NAR for HCC patients with MVI.

Study Design: A total of 362 consecutive HCC patients diagnosed with MVI after hepatic resection between February 2005 and December 2013 were included in this study. The patient outcomes were compared, and a 1:2 propensity score matching (PSM) analysis was applied to eliminate selection bias.

Results: Before PSM, compared to the NAR group, the AR group contained more patients that exceeded the Milan criteria, with larger, unilobar tumors and higher AST levels. After PSM, 100 patients were classified into the propensity-matched AR group (PS-AR), while 170 were classified into the propensity-matched NAR group (PS-NAR). Baseline data, including liver function and tumor burden measurements, were similar in the matched groups. The respective 1-, 3- and 5-year overall survival (OS) rates were $78.9 \%, 56.9 \%$, and $51.5 \%$ in the PS-AR group and $76.2 \%, 53.0 \%$, and $42.4 \%$ in the PS-NAR group $(P=0.301)$. The 1-, 3- and 5-year disease-free survival (DFS) rates were $51.1 \%$, $44.7 \%$ and $42.0 \%$ in the PS-AR group and $44.9 \%, 34.3 \%$ and $26.4 \%$ in the PS-NAR group, respectively $(P=0.039)$. Multivariate analysis identified $A R(P=0.025)$ as an independent favorable prognostic factor for DFS in HCC patients with MVI.
\end{abstract}

Conclusions: Anatomical resection was superior to non-anatomical resection for improving DFS in hepatocellular carcinoma patients with microscope vascular invasion.

Key words: Hepatocellular carcinoma, Anatomical resection, Microscope vascular invasion, Propensity score matching, Overall survival, Disease-free survival

\section{Introduction}

Hepatocellular carcinoma (HCC) is the fifth most common primary malignancy and third most common cause of cancer-related mortality worldwide, especially in Asian and African countries[1]. 
Although many treatment options exist for HCC[2], hepatic resection remains one of the proven potentially curative treatments[3]. High postoperative recurrence remains a serious problem, as recurrence occurs in the first 3 years after curative hepatic resection in approximately $50 \%-60 \%$ of cases and in the first 5 years in more than $70 \%$ of cases, even for patients with small HCCs[4, 5].

Anatomical resection (AR) may be effective for the eradication of intrahepatic microscopic metastases and prevent the development of metastasis upon the systematic removal of at least one tumor-containing Couinaud's segment, while microscopic vascular dissemination is considered the main treatment route for intrahepatic metastases[6-8]. Many studies have demonstrated that completely eradicating tumor-bearing portal tributaries may confer better overall survival (OS) and disease-free survival (DFS) than non-anatomical resection (NAR)[9-12]. On the other hand, some studies indicated no differences in HCC recurrence or OS rates between the two groups subjected to curative intent resection[13-16]. Nevertheless, these conclusions have limited the statistical power of the data because of heterogeneities in the clinical characteristics of the patients, and no studies have compared the outcomes between AR and NAR in HCC patients with microscopic vascular invasion (MVI)[17, 18]. Therefore, the advantages of AR for HCC patients with MVI remain controversial.

In this retrospective study, we investigated the prognostic outcomes of AR and NAR in HCC patients with MVI. Propensity score matching (PSM) [18, 19] was used to eliminate possible selection bias arising from the patients' backgrounds to better determine the impacts of the operative approaches on OS and DFS.

\section{Methods}

\section{Patients}

From February 2005 to December 2013, consecutive patients with HCC undergoing hepatic resection with curative intent at Sun Yat-sen University Cancer Center were evaluated for this study. All clinical data were prospectively collected in an HCC database and reviewed retrospectively.

Among these patients, only those who met all of the following inclusion criteria were selected in the study: (a) between 18 and 75 years of age with good operative tolerance, (b) had a Child-Pugh class A status, (c) had pathologically classified primary HCC, (d) had microscopic vascular invasion as determined by postoperative pathology, (e) had no anticancer treatments prior to the operation, and (f) had no evidence of extrahepatic metastasis.
Patients were excluded from the study if they met one or more of the following exclusion criteria: (a) macroscopic vascular invasion, (b) incomplete clinical data, (c) palliative hepatic resection, or (d) a history of other cancers.

\section{Diagnosis}

All patients were diagnosed with HCC according to preoperative radiographic results and laboratory tests[20]. MVI was defined as the presence of a tumor in a portal vein, hepatic vein, or large capsular vessel of the surrounding hepatic tissue lined by endothelium that was visible only by microscopy[21].

\section{Surgical procedure}

Hepatic resection was performed using previously described techniques[22, 23]. The resection methods and resection planes were chosen preoperatively at an HCC multidisciplinary team (MDT) meeting considering the patients' background variables, including their liver function, tumor location, drainage tumor area, remnant liver volume, reserved liver function and technical difficulty of liver resection. AR was defined as the systematic removal of at least one Couinaud's segment, including left/ right hemihepatectomy, left lateral lobectomy and segmental hepatectomy. Intraoperative ultrasonography was performed if necessary to confirm the number and extent of lesions and to identify the portal or hepatic vein of the tumor-bearing section. To appropriately identify segments for removal, the liver was divided according to liver surface demarcations after finding and controlling the hepatic pedicle of the targeted part of the liver under intraoperative ultrasound guidance. NAR was defined as tumor resection with a negative tumor margin without regard to Couinaud's segments or sectional or lobar structures. The Pringle maneuver was applied if necessary.

\section{Propensity score matching analysis}

Because this was a retrospective study and the operative approach was not assigned randomly, there was potential for confounding and selection biases between groups that could impact the comparisons of outcomes. To overcome the biases produced by disequilibrium between the two groups, PSM was conducted.

The propensity score was calculated by the logistical regression model using the following baseline characteristics as covariates: age, liver cirrhosis, prothrombin time (PT), platelets (PLT), ALB), total bilirubin (TBIL), aspartate transaminase (AST), Albumin-Bilirubin (ALBI) grade, alpha-fetoprotein (AFP), tumor extent, tumor size and 
Milan criteria. PSM was performed as one-to-two matching between the AR and NAR groups with nearest neighbor matching and a 0.1 caliper width using SPSS (IBM SPSS Statistics for Windows, version 19.0. IBM Corp., Armonk, NY) and Propensity Score Matching for SPSS, version 1.0 (Felix Thoemmes, Cornell University/University of Tübingen).

Table 1. Background characteristics of patients before and after propensity score matching analysis

\begin{tabular}{|c|c|c|c|c|c|c|}
\hline \multirow[t]{2}{*}{ Characteristics } & \multicolumn{3}{|c|}{ Before PSM } & \multicolumn{3}{|c|}{ After PSM } \\
\hline & $\begin{array}{l}\text { AR } \\
(n=124)\end{array}$ & $\begin{array}{l}\text { NAR } \\
(n=238)\end{array}$ & $\begin{array}{l}\text { P-valu } \\
\text { e }\end{array}$ & $\begin{array}{l}\text { PS-AR } \\
(n=100)\end{array}$ & $\begin{array}{l}\text { PS-NAR } \\
(\mathrm{n}=170)\end{array}$ & $\begin{array}{l}\text { P-valu } \\
\text { e }\end{array}$ \\
\hline \multicolumn{7}{|l|}{ Epidemiology } \\
\hline Age (year) & $46 \pm 11.5$ & $47.9 \pm 11.4$ & 0.140 & $46.9 \pm 11.5$ & $48.1 \pm 11.2$ & 0.420 \\
\hline Gender & & & 0.537 & & & 0.311 \\
\hline Male & $110(88.7)$ & $216(90.8)$ & & $88(88.0)$ & 156(91.8) & \\
\hline Female & $14(11.3)$ & $22(9.2)$ & & $12(12.0)$ & $14(8.2)$ & \\
\hline \multicolumn{7}{|l|}{ Etiology } \\
\hline $\begin{array}{c}\text { Virus(HBV/HC } \\
\text { V) }\end{array}$ & 109(87.9) & $224(94.1)$ & 0.417 & $87(87.0)$ & $158(92.9)$ & 0.697 \\
\hline Others & $15(12.1)$ & $14(5.9)$ & 0.039 & 13(13.0) & $12(7.1)$ & 0.104 \\
\hline \multicolumn{7}{|l|}{ Liver function } \\
\hline PT & $12.2 \pm 1.1$ & $12.2 \pm 1.1$ & 0.75 & $12.1 \pm 1.2$ & $12.2 \pm 1.1$ & 0.363 \\
\hline PLT $\left({ }^{*} 109 / \mathrm{L}\right)$ & $\begin{array}{l}193.6 \pm 72 . \\
4\end{array}$ & $\begin{array}{l}190.9 \pm 72 . \\
8\end{array}$ & 0.736 & $\begin{array}{l}195.7 \pm 73 . \\
6\end{array}$ & $\begin{array}{l}185.9 \pm 70 . \\
9\end{array}$ & 0.283 \\
\hline $\operatorname{ALB}(\mathrm{g} / \mathrm{L})$ & & & 0.745 & & & 0.751 \\
\hline$>35$ & $119(96.0)$ & $230(96.6)$ & & $97(97.0)$ & $162(95.3)$ & \\
\hline$\leq 35$ & $5(4.0)$ & $8(3.4)$ & & $3(3.0)$ & $8(4.7)$ & \\
\hline AST (U/L) & & & 0.015 & & & 0.175 \\
\hline$>45$ & $59(47.6)$ & $82(34.5)$ & & $43(43.0)$ & $59(34.7)$ & \\
\hline$\leq 45$ & $65(52.4)$ & $156(65.5)$ & & $57(57.0)$ & $111(65.3)$ & \\
\hline TBIL (umol/L) & & & 0.160 & & & 0.513 \\
\hline$>17.5$ & $41(33.1)$ & $62(26.1)$ & & $32(32.0)$ & $48(28.2)$ & \\
\hline$\leq 17.5$ & $83(66.9)$ & $176(73.9)$ & & $68(68.0)$ & $122(71.8)$ & \\
\hline Cirrhosis & $91(73.4)$ & $172(72.3)$ & 0.821 & $74(74.0)$ & $125(73.5)$ & 0.932 \\
\hline ALBI & & & 0.887 & & & 0.378 \\
\hline Grade 1 & $91(73.4)$ & $173(72.7)$ & & $75(75.0)$ & $119(70.0)$ & \\
\hline Grade $2 \& 3$ & $33(26.6)$ & $65(27.3)$ & & $25(25.0)$ & $51(30.0)$ & \\
\hline Milan & $29(23.4)$ & $84(35.3)$ & 0.020 & $27(27.0)$ & $54(31.8)$ & 0.409 \\
\hline \multicolumn{7}{|l|}{ Tumor burden } \\
\hline $\mathrm{AFP}(\mathrm{ng} / \mathrm{ml})$ & & & 0.595 & & & 0.688 \\
\hline$>400$ & $62(50.0)$ & 112(47.1) & & $49(49.0)$ & $79(46.5)$ & \\
\hline$\leq 400$ & $62(50.0)$ & $126(52.9)$ & & $51(51.0)$ & $91(53.5)$ & \\
\hline Tumor extent & & & 0.010 & & & 0.629 \\
\hline Unilobar & 122(98.4) & 218(91.6) & & $98(98.0)$ & 168(98.8) & \\
\hline Bilobar & $2(1.6)$ & $20(8.4)$ & & $2(2.0)$ & $2(1.2)$ & \\
\hline Tumor number & & & 0.705 & & & 0.230 \\
\hline Single & 91(73.4) & $179(75.2)$ & & $75(75.0)$ & $138(81.2)$ & \\
\hline Multiple & $33(26.6)$ & $59(24.8)$ & & $25(25.0)$ & $32(18.8)$ & \\
\hline Tumor size $(\mathrm{cm})$ & & & 0.031 & & & 0.326 \\
\hline$>5$ & $94(75.8)$ & $154(64.7)$ & & $75(75.0)$ & $118(69.4)$ & \\
\hline$\leq 5$ & $30(24.2)$ & $84(35.3)$ & & $25(25.0)$ & $52(30.6)$ & \\
\hline
\end{tabular}

Values are expressed as the mean \pm SD or no. (\%), unless otherwise indicated; PSM, propensity score matching: $A R$, anatomical resection; NAR, non-anatomical resection; PS-AR, propensity-matched anatomical resection; PS-NAR, propensity-matched non-anatomical resection; $\mathrm{HBV}$, hepatitis B virus; $\mathrm{HCV}$, hepatitis C virus; PT, prothrombin time; PLT, platelet count; ALB albumin; AST, aspartate aminotransferase; TBIL, total bilirubin; ALBI, Albumin-Bilirubin grade; Milan, within Milan criteria; AFP, alpha-fetoprotein.

\section{Postoperative follow-up}

After the liver operation, all patients were followed up by physical examination, tumor marker assessment, liver biochemistry and function assessment, blood tests, abdominal ultrasonography, and contrast-enhanced computed tomography (CT) at least every 3 months for the first year and every 6 months thereafter for more than 60 months after treatment. Complications were reported according to the Clavien-Dindo classification[24]. Recurrence was defined by findings using at least two imaging methods, such as CT and enhanced magnetic resonance imaging (MRI), and treatment for recurrent HCC was determined according to the location and number of recurrent tumors, the patient's liver function and the results of discussion among our MDT team[25].

\section{Statistical analysis}

The clinical database was established with SPSS. Continuous variables, presented as medians and ranges, were compared using Student's T-test or the Mann-Whitney U-test. Categorical data were compared using chi-squared and Fisher's exact tests. OS and DFS rates were determined using Kaplan-Meier survival curves and compared by the log-rank test. Prognostic factors identified as being significant in univariate analysis $(\mathrm{P}<0.1)$ were subjected to multivariate analysis with the Cox proportional hazards regression model. For all tests, $\mathrm{P}$ values less than 0.05 were considered statistically significant.

\section{Results}

\section{Patient characteristics}

A total of 362 patients with MVI who underwent primary resection were included in this study. The baseline characteristics of the entire study population are reported in Table 1; 124 patients undergone anatomical resection were assigned to the AR group $(34.3 \%)$, and 238 undergone non-anatomical resections were assigned to the NAR group (65.7\%). Compared to the NAR group, more patients in the AR group exceeded the Milan criteria before PSM, with larger, unilobar tumors and higher AST levels (Table 1). After the 1:2 PSM, 270 patients were recruited for comparison; 100 patients were classified into the propensity-matched AR group (PS-AR, 37\%), and 170 were classified into the propensity-matched NAR group (PS-NAR, 63\%). The background characteristics and preoperative factors of the patients in the two groups after PSM are shown in Table 1. No obvious differences were observed between the PS-AR and PS-NAR groups.

\section{Clinical outcomes}

Before PSM, the median follow-up period for all patients was 30.39 months (range, 0.2-108.0 months). For all patients, the 1-, 3- and 5-year OS rates were 
$77.0 \%, 54.0 \%$ and $46.0 \%$, respectively, and the 1-, 2and 3-year DFS rates were $45.6 \%, 37.1 \%$ and $31.4 \%$, respectively. Next, the OS and DFS curves for the two groups were stratified according to the surgical procedure employed (Fig. 1A \& 1B). The DFS rates of the AR group (1-, 2- and 3-year: 49.8\%, 44.2\%, 40.7\%, respectively) were better than those of the NAR group (1-, 2- and 3-year: $44.0 \%, 34.2 \%, 27.7 \%$, respectively, $\mathrm{P}=0.045$, Fig. 1B). By contrast, the OS rates of the AR group were not statistically different from those of the NAR group (AR vs. NAR: $76.5 \%, 54.4 \%, 48.1 \%$ vs. $77.2 \%, 53.8 \%, 44.7 \%$, respectively, $\mathrm{P}=0.645$, Fig. $1 \mathrm{~A}$ ).

After PSM, the median follow-up period was 31.46 months (range, 0.23-136.74 months). The 1-, 3and 5-year OS rates were $77.2 \%, 54.5$ and $46.1 \%$, respectively, and the 1-, 2- and 3-year DFS rates were $46.5 \%, 37.7 \%$ and $31.9 \%$, respectively. The OS and DFS curves for the two groups (PS-AR vs. PS-NAR) were stratified according to the surgical procedure employed (Fig.2A \& 2B). For the PS-AR group, the respective $1-, 3$ - and 5-year OS rates were $78.9 \%$, $56.9 \%$, and $51.5 \%$, and the median survival time was 33.4 months. For the PS-NAR group, the respective 1-, 3 -, and 5-year OS rates were $76.2 \%, 53.0 \%$, and $42.4 \%$, and the median survival time was 30.6 months $(\mathrm{P}=0.301$, Fig. 2A). Significant differences existed between the DFS rates of the PS-AR and PS-NAR groups ( $\mathrm{P}=0.039$, Fig. $2 \mathrm{~B})$. The 1-, 2- and 3- DFS rates and median DFS time were $51.1 \%, 44.7 \%, 42.0 \%$, and 12.0 months, respectively, for the PS-AR group and $44.9 \%, 34.3 \%, 26.4 \%$ and 10.1 months, respectively, for the PS-NAR group.

\section{Surgical variables and postoperative complications}

Table 2 lists the surgery-related variables, tumor variables, postoperative complications and types of recurrence between the AR and NAR groups. Although the patients who underwent AR showed a greater portion of major resection (39\% vs. $18.8 \%$, $\mathrm{P}=0.000)$ and a longer operation time $(186.7 \pm 63.0 \mathrm{~min}$
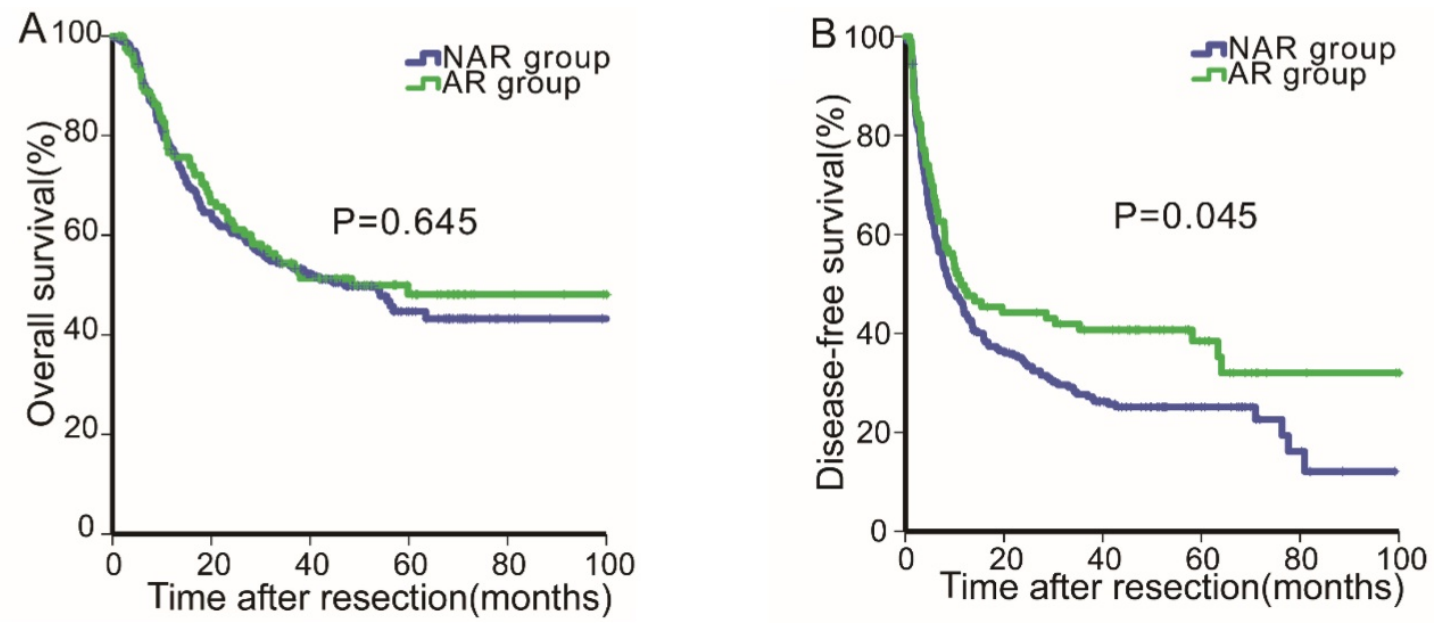

Figure 1. Overall survival (1A) and disease-free survival (1B) curves of patients in the anatomical
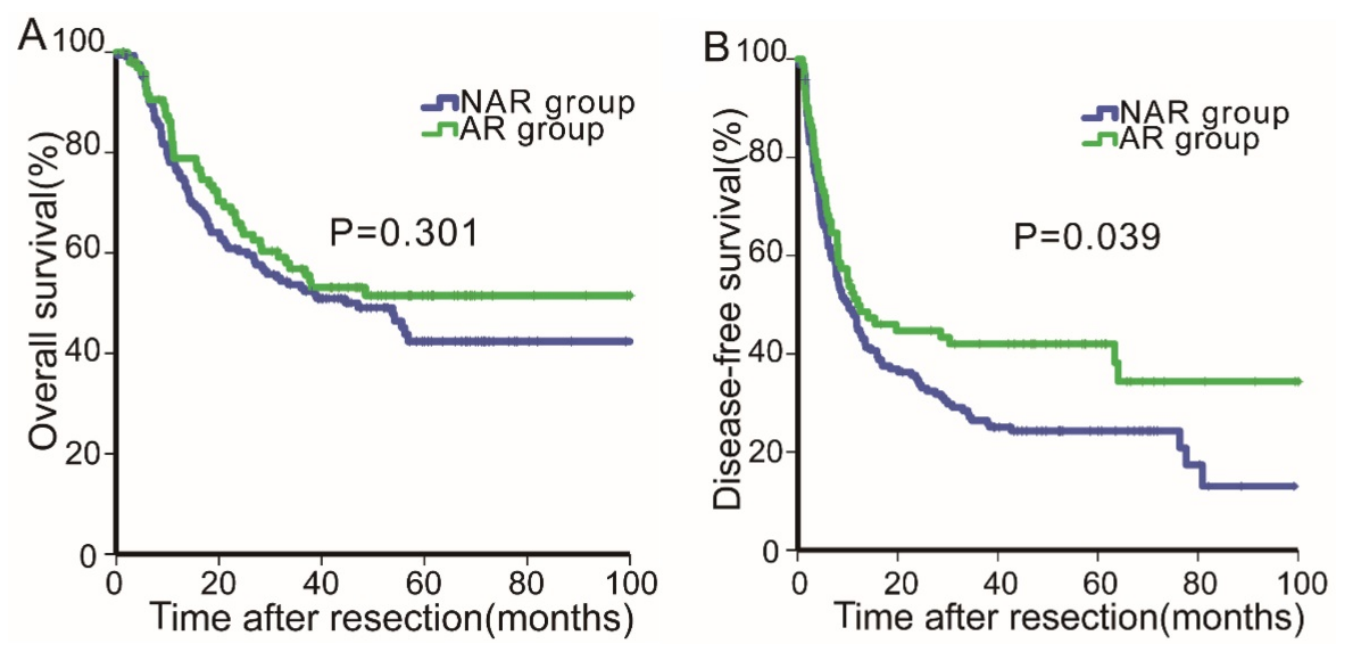

Figure 2. Overall survival (2A) and disease-free survival (2B) curves of patients in the anatomical resection and non-anatomical resection groups after propensity score matching analysis. 
vs. $171.8 \pm 50.8 \mathrm{~min}, \mathrm{P}=0.043$ ) than those who underwent NAR, the postoperative complications $(\mathrm{P}=0.499)$, blood loss $(552.3 \pm 1076.1 \mathrm{ml}$ vs. $457.6 \pm 452.7$ $\mathrm{ml}, \mathrm{P}=0.315)$ and time of the Pringle maneuver $(10.5 \pm 13.2$ vs. $14.0 \pm 11.2, \quad \mathrm{P}=0.060)$ were not significantly different between the two group before and after PSM.

\section{Univariate and multivariate analyses of overall survival and disease-free survival}

Univariate and multivariate analyses were performed to identify the predictors that influenced OS and DFS both before and after PSM, and the results are shown in Tables 3 and 4. Before matching, univariate analysis revealed that the PT, AST, ALBI grade, Milan criteria, AFP, tumor extent, tumor number and tumor size factors influenced the OS rates, while multivariate analysis identified PT $(\mathrm{P}=0.03)$, AST $(\mathrm{P}=0.034)$, TBIL $(\mathrm{P}=0.022)$ and tumor number $(\mathrm{P}=0.001)$ as independent risk factors. Additionally, the PT, AST, ALBI grade, Milan criteria, $\mathrm{AFP}$, tumor extent, tumor number, tumor size and AR factors affected the DFS rates according to univariate analysis, and multiple analysis indicated that PT $(\mathrm{P}=0.008)$, AFP $(\mathrm{P}=0.020)$, multiple tumors $(\mathrm{P}=0.003)$ and $\mathrm{AR}(\mathrm{P}=0.009)$ were independent prognostic factors for DFS.

Table 4 shows the factors (PT, AST, liver cirrhosis, ALBI grade, Milan criteria, tumor extent, tumor number and tumor size) that influenced the OS rates of MVI patients after PSM. These analyses also indicated that the DFS rates were significantly related to the PT, serum album, ALBI grade, Milan criteria, tumor extent, tumor number, tumor size and AR factors. Multivariate analysis identified that PT $(\mathrm{P}=0.001)$ and multiple tumors $(\mathrm{P}=0.001)$ independently influenced the OS rates. PT $(\mathrm{P}=0.000)$, album $(\mathrm{P}=0.031)$, AFP (0.008), bilobar tumor extent $(P=0.004)$, multiple tumors $(P=0.019)$ and $A R$ $(\mathrm{P}=0.025)$ were independent unfavorable prognostic factors for DFS in HCC patients.

\section{Discussion}

In this retrospective study, the clinical outcomes of $A R$ and NAR performed with curative intent on HCC patients with MVI were investigated using PSM analysis. Furthermore, our study indicated that AR performed on HCC patients with MVI provided better DFS rates after initial resection. Univariate and multivariate analyses indicated that $A R$ was an independent favorable prognostic factor for DFS.

Table 2. Surgery-related characteristics and postoperative outcome

\begin{tabular}{|c|c|c|c|c|c|c|}
\hline \multirow[t]{2}{*}{ Characteristics } & \multicolumn{3}{|c|}{ Before PSM } & \multicolumn{3}{|c|}{ After PSM } \\
\hline & AR (n=124) & NAR $(n=238)$ & P-value & PS-AR (n=100) & PS-NAR (n=170) & P-value \\
\hline \multicolumn{7}{|l|}{ Surgical variables } \\
\hline Surgical margin $(\geq 1 \mathrm{~cm})$ & $77(62.1)$ & $125(52.5)$ & 0.082 & $64(64)$ & $91(53.5)$ & 0.093 \\
\hline Scope of resection & & & 0.000 & & & 0.000 \\
\hline Major resection & $51(41.1)$ & $52(21.8)$ & & $39(39)$ & $32(18.8)$ & \\
\hline Minor resection & $73(58.9)$ & $186(78.2)$ & & $61(61)$ & $138(81.2)$ & \\
\hline Operation time (min) & $191.4 \pm 65.1$ & $170.3 \pm 47.6$ & 0.002 & $186.7 \pm 63.0$ & $171.8 \pm 50.8$ & 0.043 \\
\hline Time of Pringle's maneuver & $10.4 \pm 13.3$ & $13.8 \pm 10.8$ & 0.015 & $10.5 \pm 13.2$ & $14.0 \pm 11.2$ & 0.060 \\
\hline Blood loss (ml) & $568.0 \pm 1001.3$ & $437.8 \pm 430.3$ & 0.169 & $552.3 \pm 1076.1$ & $457.6 \pm 452.7$ & 0.315 \\
\hline \multicolumn{7}{|l|}{ Tumor variables } \\
\hline Single tumor & $91(73.4)$ & $179(75.2)$ & 0.705 & $75(75.0)$ & $138(81.2)$ & 0.230 \\
\hline Tumor size $>5(\mathrm{~cm})$ & $94(75.8)$ & $154(64.7)$ & 0.031 & $75(75.0)$ & $118(69.4)$ & 0.326 \\
\hline Capsule (with) & $83(66.9)$ & $153(64.3)$ & 0.615 & $67(67)$ & $117(68.8)$ & 0.756 \\
\hline Edmondson grades" & & & 0.248 & & & 0.183 \\
\hline I, II & $75(60.5)$ & $159(66.8)$ & & $62(62.0)$ & $119(70.0)$ & \\
\hline III, IV & $49(39.5)$ & $79(33.2)$ & & $38(38.0)$ & $51(30.0)$ & \\
\hline Complications* & & & 0.279 & & & 0.499 \\
\hline I & $60(48.4)$ & $119(50.0)$ & & $39(39.0)$ & $59(34.7)$ & \\
\hline II & $32(25.8)$ & 75 (31.5) & & $25(25.0)$ & $52(30.6)$ & \\
\hline III & $27(21.8)$ & $40(16.8)$ & & $31(31.0)$ & $55(32.4)$ & \\
\hline IV & $5(4.0)$ & $4(1.7)$ & & $5(5.0)$ & $4(2.4)$ & \\
\hline $\mathrm{V}$ & 0 & 0 & & 0 & 0 & \\
\hline \multicolumn{7}{|l|}{ Recurrence } \\
\hline Intrahepatic recurrence & $65(52.4)$ & $123(51.7)$ & 0.498 & $51(51.0)$ & $87(51.2)$ & 0.978 \\
\hline Extrahepatic metastasis & $32(25.8)$ & $54(22.7)$ & 0.508 & $24(24.0)$ & $45(26.5)$ & 0.653 \\
\hline Vascular invasion & $13(10.5)$ & $21(8.8)$ & 0.607 & $10(10.0)$ & $18(10.6)$ & 0.878 \\
\hline
\end{tabular}


Table 3. Univariate analysis and multivariate analysis of overall survival and disease-free survival for patients before propensity matching analysis.

\begin{tabular}{|c|c|c|c|c|c|c|c|c|}
\hline \multirow[t]{3}{*}{ Variables } & \multicolumn{4}{|c|}{ OS } & \multicolumn{4}{|c|}{ DFS } \\
\hline & \multirow{2}{*}{$\begin{array}{l}\text { Univariate analysis } \\
\text { P-value }\end{array}$} & \multicolumn{3}{|c|}{ Multivariate analysis } & \multirow{2}{*}{$\begin{array}{l}\text { Univariate analysis } \\
\text { P-value }\end{array}$} & \multicolumn{3}{|c|}{ Multivariate analysis } \\
\hline & & HR & $95 \% \mathrm{CI}$ & P-value & & HR & $95 \% \mathrm{CI}$ & P-value \\
\hline Age $(\leq 50 />50)$ (year) & 0.832 & & & & 0.643 & & & \\
\hline Gender (male/female) & 0.247 & & & & 0.528 & & & \\
\hline Virus ( positive/negative) & 0.475 & & & & 0.176 & & & \\
\hline $\mathrm{PT}(\leq 13.5 />13.5)(\mathrm{sec})$ & 0.000 & 1.976 & $1.262-3.094$ & 0.03 & 0.001 & 1.756 & $1.160-2.659$ & 0.008 \\
\hline $\operatorname{PLT}(\leq 100 />100)\left({ }^{*} 109 / \mathrm{L}\right)$ & 0.924 & & & & 0.816 & & & \\
\hline $\operatorname{ALB}(\leq 35 />35)(\mathrm{g} / \mathrm{L})$ & 0.217 & & & & 0.234 & & & \\
\hline AST $(\leq 45 />45)(\mathrm{U} / \mathrm{L})$ & 0.000 & 1.420 & $1.027-1.965$ & 0.034 & 0.048 & & & \\
\hline TBIL $(\leq 17.5 />17.5)(\mathrm{umol} / \mathrm{L})$ & 0.062 & 1.494 & $1.059-2.110$ & 0.022 & 0.329 & & & \\
\hline Cirrhosis (yes/no) & 0.211 & & & & 0.557 & & & \\
\hline ALBI ( grade 1/grade $2 \& 3$ ) & 0.020 & & & & 0.024 & & & \\
\hline Milan & 0.000 & & & & 0.000 & & & \\
\hline $\operatorname{AFP}(\leq 400 />400)(\mathrm{ng} / \mathrm{ml})$ & 0.036 & & & & 0.011 & 1.367 & $1.051-1.778$ & 0.020 \\
\hline Tumor extent (unilobar/bilobar) & 0.023 & & & & 0.047 & & & \\
\hline Tumor number (single/multiple) & 0.000 & 1.809 & $1.274-2.568$ & 0.001 & 0.000 & 1.592 & $1.166-2.174$ & 0.003 \\
\hline Tumor size $(\leq 5 />5)(\mathrm{cm})$ & 0.000 & & & & 0.000 & & & \\
\hline Operative procedure (AR/NAR) & 0.645 & & & & 0.045 & 1.504 & $1.105-2.045$ & 0.009 \\
\hline
\end{tabular}

OS, overall survival; DFS, disease-free survival; HR, hazard ratio; $\mathrm{CI}$, confidence interval; $\mathrm{AR}$, anatomical resection; NAR, non-anatomical resection; $\mathrm{PT}$, prothrombin time; PLT, platelet count; ALB, albumin; AST, aspartate aminotransferase; TBIL, total bilirubin; ALBI, Albumin-Bilirubin grade; Milan, within Milan criteria; AFP, alpha-fetoprotein.

Table 4. Univariate analysis and multivariate analysis of overall survival and disease-free survival for patients after propensity matching analysis.

\begin{tabular}{|c|c|c|c|c|c|c|c|c|}
\hline \multirow[t]{3}{*}{ Variables } & \multicolumn{4}{|c|}{ OS } & \multicolumn{4}{|c|}{ DFS } \\
\hline & \multirow{2}{*}{$\begin{array}{l}\text { Univariate analysis } \\
\text { P-value }\end{array}$} & \multicolumn{3}{|c|}{ Multivariate analysis } & \multirow{2}{*}{$\begin{array}{l}\text { Univariate analysis } \\
\text { P-value }\end{array}$} & \multicolumn{3}{|c|}{ Multivariate analysis } \\
\hline & & HR & 95\%CI & P-value & & HR & $95 \% \mathrm{CI}$ & P-value \\
\hline Age $(\leq 50 />50)$ (year) & 0.914 & & & & 0.243 & & & \\
\hline Gender (male/female) & 0.252 & & & & 0.923 & & & \\
\hline Virus ( positive/negative) & 0.904 & & & & 0.487 & & & \\
\hline $\mathrm{PT}(\leq 13.5 />13.5)(\mathrm{sec})$ & 0.000 & 2.475 & $1.475-4.153$ & 0.001 & 0.001 & 2.410 & $1.486-3.909$ & 0.000 \\
\hline $\operatorname{PLT}(\leq 100 />100)\left({ }^{*} 109 / \mathrm{L}\right)$ & 0.899 & & & & 0.996 & & & \\
\hline $\operatorname{ALB}(\leq 35 />35)(\mathrm{g} / \mathrm{L})$ & 0.182 & & & & 0.041 & 2.224 & $1.076-4.595$ & 0.031 \\
\hline $\operatorname{AST}(\leq 45 />45)(\mathrm{U} / \mathrm{L})$ & 0.018 & & & & 0.176 & & & \\
\hline TBIL $(\leq 17.5 />17.5)(\mathrm{umol} / \mathrm{L})$ & 0.217 & & & & 0.766 & & & \\
\hline Cirrhosis (yes/no) & 0.043 & & & & 0.534 & & & \\
\hline ALBI ( grade 1 /grade $2 \& 3$ ) & 0.096 & & & & 0.081 & & & \\
\hline Milan & 0.003 & & & & 0.000 & & & \\
\hline $\operatorname{AFP}(\leq 400 />400)(\mathrm{ng} / \mathrm{ml})$ & 0.123 & & & & 0.031 & 1.510 & $1.113-2.050$ & 0.008 \\
\hline Tumor extent (unilobar/bilobar) & 0.021 & & & & 0.000 & 5.963 & $1.762-20.174$ & 0.004 \\
\hline Tumor number (single/multiple) & 0.000 & 1.976 & $1.323-2.951$ & 0.001 & 0.003 & 1.560 & $1.076-2.262$ & 0.019 \\
\hline Tumor size $(\leq 5 />5)(\mathrm{cm})$ & 0.004 & & & & 0.000 & & & \\
\hline Operative procedure (AR/NAR) & 0.301 & & & & 0.039 & 1.477 & $1.049-2.079$ & 0.025 \\
\hline
\end{tabular}

HCC with MVI has a high incidence of recurrence and metastasis via the intrahepatic portal vein system, which is the main reason for its bad prognosis, [26, 27] and MVI has been reported as a risk survival factor following resection. Survival chances are improved by eliminating macroscopic and microscopic liver metastases and preserving functional liver parenchymal cells to the greatest extent possible. Therefore, AR is a theoretically ideal procedure, as it may remove the portal tributaries bearing the tumor completely and reduce the ischemic operation area[28, 29]. In clinical practice, surgeons often choose AR for treating patients with good liver function, small tumors and no cirrhosis to reduce the risks in terms of difficulty of the $\mathrm{AR}$ technique and remnant liver functions[19, 30]. Indeed, our study showed significantly longer operation times for the AR procedure. The postoperative complications, intraoperative blood loss and rates of homologous blood transfusion were similar between the AR and NAR groups.

Previous studies compared the clinical outcomes of HCC patients subjected to the AR and NAR procedures[17, 31, 32]. Zhou et al.[11], Eguchi et al.[12] and Hasegawa et al.[33] concluded that AR was superior to NAR for prolonging OS and DFS after hepatic resection. However, other researchers reported different findings[16, 31]. Marubashi et 
al.[18] proposed that the OS and DFS rates in the AR and NAR groups were not statistically different. Chen et al.[30] and Zhao et al.[17] reported that AR contributed to better DFS rates, but the OS rates between the AR and NAR groups were similar. Whether AR is superior to NAR remains controversial, and data on the effects of performing AR on HCC patients with MVI are lacking. This study confirmed that AR resulted in better DFS rates than NAR. Though the Kaplan-Meier survival curves of OS were not significantly different between the AR and NAR groups, the 5-year survival rates in the AR group were higher than those in the NAR group both before and after PSM analysis $(48.1 \%$ vs. $44.7 \%$ and $51.5 \%$ vs. $42.4 \%$, respectively).

In the original unmatched group, the short-term OS rates were similar between patients undergoing AR and NAR (1-, 3-year: $76.5 \%, 54.4 \%$ vs. $77.2 \%$, $53.8 \%$, respectively). The AR group included a higher proportion of patients with large tumors, multiple tumors, liver cirrhosis and high AFP levels than the NAR group. These were all known risk factors associated with tumor recurrence and reduced survival, which might explain why the short-term outcomes were nearly the same between the two groups[34, 35]. After using 1:2 PSM analysis to reduce selection bias, the 1-, 3- and 5-year OS rates in the PS-AR group were higher than those in the PS-NAR group $(78.9 \%, 56.9 \%$, and $51.5 \%$ vs. $76.2 \%, 53.0 \%$, and 42. $4 \%$, respectively). When comparing AR and NAR patients, the differences in the long-term survival rates were more deviated than those in the short-term survival rates. In summary, AR patients have better long-term survival prognoses than NAR patients.

In this retrospective study, a 1:2 propensity score matching (PSM) analysis was used to eliminate possible selection biases between two groups. As a result, selection biases were significantly reduced but not completely eliminated which could impact the clinical outcomes. In the AR cohort, some patients underwent hemihepatectomy with multiple tumors $(26.6 \%$ vs. $24.8 \%)$ and larger tumor sizes $(8.1 \pm 3.7 \mathrm{~cm}$ vs. $6.6 \pm 3.7 \mathrm{~cm}, \mathrm{P}<0.000)$. Although PSM was conducted, the disequilibrium between groups still existed (multiple tumor $25.0 \%$ vs. $18.8 \%$, tumor sizes $7.8 \pm 3.7 \mathrm{~cm}$ vs. $7.1 \pm 3.6 \mathrm{~cm}$ ), which might have impacts on the clinical outcomes. However, anatomical resection may systematic removed microscopic metastases and prevent metastasis. Therefore, the OS of AR-group did not find significant advantage, but DFS was significantly better than that of control group, and the 5 -year OS rates was better $(51.5 \%$ vs. 42. $4 \%$ ). In conclusion, anatomical hepatectomy may lead to better clinical outcomes for HCC patients with MVI.
This study did have several limitations. First, the research was designed as a single-center, retrospective, and non-randomized controlled study. The operative procedures were decided upon by various clinical doctors in the MDT group. Even if PSM was used to limit the number of similar baseline characteristics, possible selection biases between the groups remained. Therefore, multicenter, randomized controlled trials are required to confirm the role of $A R$ in HCC patients with MVI.

In conclusion, our study indicated that AR improved the RFS rates in patients with MVI. If a surgical procedure is feasible and preoperative examination shows the possibility of MVI, AR is recommended.

\section{Conclusions}

In the present study, we aimed to investigate the prognostic outcomes of AR and NAR for HCC patients with MVI. PSM was used to eliminate possible selection bias arising from the patients' backgrounds to better determine the impacts of the operative approaches on OS and DFS.

As a result, we found that AR was superior to NAR for improving DFS in HCC patients with MVI. Indeed, the 5-year survival rates in the AR group were higher than those in the NAR group.

\section{Acknowledgements}

The authors would like to thank Dr. Ling Yi-hong and Pro. Shi Ming of the State Key Laboratory of Oncology in South China (Guangzhou, China) for their help during the experiment.

\section{Funding}

This study was supported by Science and Technology Planning Project of Guangdong Province, China (2017A020215112), Natural Science Foundation of Guangdong Province, China (2017A030310203), Shanghai Sailing Program (No. 18YF1404900), Fudan University Shanghai Cancer Center hospital-level research fund (No.YJ201709) and the Ethicon "EXCELLENCE IN SURGERY" Grant (No. 320.2710.1823).

\section{Author Contributions}

ZYF and ZXP designed the study. The article is written by ZXP and WW. M J and KA collected the patients' samples. LLH and LSH performed the statistical analysis. Professor Guo and Chen are responsible for guidance. All authors read and approved the final manuscript.

\section{Competing Interests}

The authors have declared that no competing interest exists. 


\section{References}

1. Torre LA, Bray F, Siegel RL, Ferlay J, Lortet-Tieulent J, Jemal A. Global cancer statistics, 2012. CA: a cancer journal for clinicians. 2015; 65: 87-108.

2. Simonetti RG, Liberati A, Angiolini C, Pagliaro L. Treatment of hepatocellular carcinoma: a systematic review of randomized controlled trials. Annals of oncology : official journal of the European Society for Medical Oncology / ESMO. 1997; 8: 117-36.

3. Llovet JM, Zucman-Rossi J, Pikarsky E, Sangro B, Schwartz M, Sherman M, et al. Hepatocellular carcinoma. Nature reviews Disease primers. 2016; 2: 16018.

4. Colecchia A, Schiumerini R, Cucchetti A, Cescon M, Taddia M, Marasco G, et al. Prognostic factors for hepatocellular carcinoma recurrence. World journal of gastroenterology. 2014; 20: 5935-50.

5. Roayaie S, Obeidat K, Sposito C, Mariani L, Bhoori S, Pellegrinelli A, et al. Resection of hepatocellular cancer $</=2 \mathrm{~cm}$ : results from two Western centers. Hepatology. 2013; 57: 1426-35.

6. Yamamoto M, Takasaki K, Ohtsubo T, Katsuragawa H, Fukuda C, Katagiri S. Effectiveness of systematized hepatectomy with Glisson's pedicle transection at the hepatic hilus for small nodular hepatocellular carcinoma: retrospective analysis. Surgery. 2001; 130: 443-8.

7. Makuuchi M, Hasegawa H, Yamazaki S. Ultrasonically guided subsegmentectomy. Surgery, gynecology \& obstetrics. 1985; 161: 346-50.

8. Sasaki K, Matsuda M, Ohkura Y, Hashimoto M, Watanabe G. Anatomical versus nonanatomical resection in patients with hepatocellular carcinoma located in the left lateral segment. The American surgeon. 2013; 79: 1163-70.

9. Shindoh J, Makuuchi M, Matsuyama Y, Mise Y, Arita J, Sakamoto Y, et al. Complete removal of the tumor-bearing portal territory decreases local tumor recurrence and improves disease-specific survival of patients with hepatocellular carcinoma. Journal of hepatology. 2016; 64: 594-600.

10. Ishii M, Mizuguchi $\mathrm{T}$, Kawamoto $\mathrm{M}$, Meguro M, Ota $\mathrm{S}$, Nishidate $\mathrm{T}$, et al, Propensity score analysis demonstrated the prognostic advantage of anatomical liver resection in hepatocellular carcinoma. World journal of gastroenterology. 2014; 20: 3335-42.

11. Zhou Y, Xu D, Wu L, Li B. Meta-analysis of anatomic resection versus nonanatomic resection for hepatocellular carcinoma. Langenbeck's archives of surgery. 2011; 396: 1109-17.

12. Eguchi S, Kanematsu $\mathrm{T}$, Arii S, Okazaki M, Okita $\mathrm{K}$, Omata $\mathrm{M}$, et al Comparison of the outcomes between an anatomical subsegmentectomy and a non-anatomical minor hepatectomy for single hepatocellular carcinomas based on a Japanese nationwide survey. Surgery. 2008; 143: 469-75

13. Hirokawa F, Kubo S, Nagano H, Nakai T, Kaibori M, Hayashi M, et al. Do patients with small solitary hepatocellular carcinomas without macroscopically vascular invasion require anatomic resection? Propensity score analysis. Surgery. 2015; 157: 27-36.

14. Marubashi S, Gotoh K, Akita H, Takahashi H, Sugimura K, Miyoshi N, et al. Analysis of Recurrence Patterns After Anatomical or Non-anatomical Resection for Hepatocellular Carcinoma. Annals of surgical oncology. 2015; 22: 2243-52.

15. Okamura Y, Ito T, Sugiura T, Mori K, Uesaka K. Anatomic versus nonanatomic hepatectomy for a solitary hepatocellular carcinoma : a case-controlled study with propensity score matching. Journal of gastrointestinal surgery : official journal of the Society for Surgery of the Alimentary Tract. 2014; 18: 1994-2002.

16. Tomimaru $\mathrm{Y}$, Eguchi $\mathrm{H}$, Marubashi $\mathrm{S}$, Wada $\mathrm{H}$, Kobayashi $\mathrm{S}$, Tanemura $\mathrm{M}$, et al. Equivalent outcomes after anatomical and non-anatomical resection of small hepatocellular carcinoma in patients with preserved liver function. Digestive diseases and sciences. 2012; 57: 1942-8.

17. Zhao H, Chen C, Gu S, Yan X, Jia W, Mao L, et al. Anatomical versus non-anatomical resection for solitary hepatocellular carcinoma without macroscopic vascular invasion: A propensity score matching analysis. Journal of gastroenterology and hepatology. 2017; 32: 870-8.

18. Marubashi S, Gotoh K, Akita H, Takahashi H, Ito Y, Yano M, et al. Anatomical versus non-anatomical resection for hepatocellular carcinoma. The British journal of surgery. 2015; 102: 776-84

19. Cucchetti A, Qiao GL, Cescon M, Li J, Xia Y, Ercolani G, et al. Anatomic versus nonanatomic resection in cirrhotic patients with early hepatocellular carcinoma. Surgery. 2014; 155: 512-21.

20. Bruix J, Sherman M, American Association for the Study of Liver D. Management of hepatocellular carcinoma: an update. Hepatology. 2011; 53: 1020-2.

21. Iguchi T, Shirabe $K$, Aishima S, Wang H, Fujita N, Ninomiya M, et al. New Pathologic Stratification of Microvascular Invasion in Hepatocellular Carcinoma: Predicting Prognosis After Living-donor Liver Transplantation. Transplantation. 2015; 99: 1236-42.

22. Zhang YF, Zhou J, Wei W, Zou RH, Chen MS, Lau WY, et al Intermediate-stage hepatocellular carcinoma treated with hepatic resection: the NSP score as an aid to decision-making. British journal of cancer. 2016; 115: 1039-47.

23. Shi M, Guo RP, Lin XJ, Zhang YQ, Chen MS, Zhang CQ, et al. Partial hepatectomy with wide versus narrow resection margin for solitary hepatocellular carcinoma: a prospective randomized trial. Annals of surgery. 2007; 245: 36-43.

24. Clavien PA, Barkun J, de Oliveira ML, Vauthey JN, Dindo D, Schulick RD, et al. The Clavien-Dindo classification of surgical complications: five-year experience. Annals of surgery. 2009; 250: 187-96.
25. Zhang YF, Guo RP, Zou RH, Shen JX, Wei W, Li SH, et al. Efficacy and safety of preoperative chemoembolization for resectable hepatocellular carcinoma with portal vein invasion: a prospective comparative study. European radiology. 2016; 26: 2078-88.

26. Shirabe K, Kajiyama K, Harimoto N, Masumoto H, Fukuya T, Ooya M, et al. Prognosis of hepatocellular carcinoma accompanied by microscopic portal vein invasion. World journal of gastroenterology. 2009; 15: 2632-7.

27. Cucchetti A, Zanello M, Cescon M, Ercolani G, Del Gaudio M, Ravaioli M, et al. Improved diagnostic imaging and interventional therapies prolong survival after resection for hepatocellular carcinoma in cirrhosis: the university of bologna experience over 10 years. Annals of surgical oncology. 2011; 18: 1630-7.

28. Matsumoto T, Kubota K, Aoki T, Iso Y, Kato M, Shimoda M. Clinical Impact of Anatomical Liver Resection for Hepatocellular Carcinoma with Pathologically Proven Portal Vein Invasion. World journal of surgery. 2016; 40: 402-11.

29. Kudo A, Tanaka S, Ban D, Matsumura S, Irie T, Nakamura N, et al. Anatomic resection reduces the recurrence of solitary hepatocellular carcinoma $</=5 \mathrm{~cm}$ without macrovascular invasion. American journal of surgery. 2014; 207: 863-9.

30. Chen J, Huang K, Wu J, Zhu H, Shi Y, Wang Y, et al. Survival after anatomic resection versus nonanatomic resection for hepatocellular carcinoma: a meta-analysis. Digestive diseases and sciences. 2011; 56: 1626-33.

31. Li SQ, Huang T, Shen SL, Hua YP, Hu WJ, Kuang M, et al. Anatomical versus non-anatomical liver resection for hepatocellular carcinoma exceeding Milan criteria. The British journal of surgery. 2017; 104: 118-27.

32. Arii S, Tanaka S, Mitsunori Y, Nakamura N, Kudo A, Noguchi N, et al. Surgical strategies for hepatocellular carcinoma with special reference to anatomical hepatic resection and intraoperative contrast-enhanced ultrasonography. Oncology. 2010; 78 Suppl 1: 125-30.

33. Hasegawa K, Kokudo N, Imamura H, Matsuyama Y, Aoki T, Minagawa M, et al. Prognostic impact of anatomic resection for hepatocellular carcinoma. Annals of surgery. 2005; 242: 252-9.

34. Vitale A, Burra P, Frigo AC, Trevisani F, Farinati F, Spolverato G, et al. Survival benefit of liver resection for patients with hepatocellular carcinoma across different Barcelona Clinic Liver Cancer stages: a multicentre study. Journal of hepatology. 2015; 62: 617-24.

35. Sumie S, Nakashima O, Okuda K, Kuromatsu R, Kawaguchi A, Nakano M, et al. The significance of classifying microvascular invasion in patients with hepatocellular carcinoma. Annals of surgical oncology. 2014; 21: 1002-9. 